\title{
Preliminary investigation of flexibility in learning color-reward associations in gibbons (Hylobatidae)
}

Justin D'Agostino and Clare Cunningham

This is the peer reviewed version of the following article:

D'Agostino, J. and Cunningham, C.L. 2015. Preliminary investigation of flexibility in learning color-reward associations in gibbons (Hylobatidae). American Journal of Primatology. 77(8): pp.854-868. Available from DOI: http://dx.doi.org/10.1002/ ajp. 22410

which has been published in final form at http://dx.doi.org/10.1002/ajp.22410. This article may be used for non-commercial purposes in accordance with Wiley Terms and Conditions for self-archiving. 
1 Preliminary investigation of flexibility in learning color-reward

2 associations in gibbons (Hylobatidae)

6 Justin D’Agostino' - California State University, Los Angeles - 5151 State University Dr Los

7 Angeles, CA 90032 - Anthropology Department - KHC 4068 - jdagost@calstatela.edu

8

9 Clare Cunningham - Evolutionary and Biological Approaches to Behaviour Research

10 Group, Department of Psychology, University of Abertay, Dundee DD1 1HG, UK

11

12

13

14

15

16

17

18

$19{ }^{1}$ Corresponding author

20

21

22

23 
D’Agostino and Cunningham 2

\section{ABSTRACT}

25 Previous studies in learning set formation have shown that most animal species can learn

26 to learn with subsequent novel presentations being solved in fewer presentations than when they

27 first encounter a task. Gibbons (Hylobatidae) have generally struggled with these tasks and do

28 not show the learning to learn pattern found in other species. This is surprising given their

29 phylogenetic position and level of cortical development. However, there have been conflicting

30 results with some studies demonstrating higher level learning abilities in these small apes. This

31 study attempts to clarify whether gibbons can in fact use knowledge gained during one learning

32 task to facilitate performance on a similar, but novel problem that would be a precursor to

33 development of a learning set. We tested 16 captive gibbons' ability to associate color cues with

34 provisioned food items in two experiments where they experienced a period of learning followed

35 by experimental trials during which they could potentially use knowledge gained in their first

36 learning experience to facilitate solution I subsequent novel tasks. Our results are similar to most

37 previous studies in that there was no evidence of gibbons being able to use previously acquired

38 knowledge to solve a novel task. However, once the learning association was made, the gibbons

39 performed well above chance. We found no differences across color associations, indicating

40 learning was not affected by the particular color / reward association. However, there were

41 variations in learning performance with regard to genera. The hoolock (Hoolock leuconedys) and

42 siamang (Symphalangus syndactylus) learned the fastest and the lar group (Hylobates sp.)

43 learned the slowest. We caution these results could be due to the small sample size and because

44 of the captive environment in which these gibbons were raised. However, it is likely that

45 environmental variability in the native habitats of the subjects tested could facilitate the 
46 evolution of flexible learning in some genera. Further comparative study is necessary in order to

47 incorporate realistic cognitive variables into foraging models.

48 INTRODUCTION

49 Despite being the most diverse group of extant apes, gibbons continue to be the most

50 understudied of all higher primates, particularly with regard to their cognitive abilities. Gibbons

51 are native to South East Asia and can be found in Vietnam, Cambodia, Thailand, Burma, China,

52 Malaysia, and the islands of Indonesia. They are omnivores and in ideal resource availability

53 conditions, high-energy fruit constitutes the majority of their diet with the remainder consisting

54 of leaves, flowers, seeds, tree bark, insects, small birds, eggs and tender plant shoots [Curran \&

55 Leighton, 2000]. These small arboreal apes primarily live in monogamous pairs with their

56 associated offspring and are characterized by limited sexual dimorphism, complex vocal duets

57 between the male and female bonded pair and brachiating mode of locomotion [Cunningham \&

58 Mootnick, 2009].

59 Representing an interesting evolutionary divergence between monkeys and great apes, a

60 better understanding of gibbon learning ability and their capacity for complex mental processes

61 would allow us to track both the progression of advanced cognition and the evolutionary

62 pressures that have led to the emergence of abilities characteristic of great apes (including

63 humans). This research therefore aims to investigate whether gibbons are able to learn sequential

64 color-reward associations and if they can extract relevant information from the first stimulus-

65 response association to enable them to make future associations more readily.

66 Research on gibbon vision has shown there are separate M and L photo pigments and

67 other characteristics that are almost identical to those of the common chimpanzee (Pan

68 troglodytes) - known to have trichromatic color vision, suggesting their searching behavior is 
69 heavily guided by vision. As all genera of gibbon enjoy the benefits of full trichromatic color

70 vision [Deegan \& Jacobs, 2001; Jacobs, 1993; Jacobs, et al., 1996], they should easily

71 discriminate color information from the cues provided during this series of studies.

72 Experimental tests of associative learning in non-human primates in a controlled setting

73 typically employ object discrimination tasks where the subject is presented with two arbitrary

74 shapes, one that if chosen will lead to a reward and one that will not. For example, selection of

75 the red square would lead to reinforcement whereas selecting the blue triangle presented with it

76 in a two-way choice paradigm would leave the subject unreinforced. Data are collected on the

77 number of trials needed to reach a pre-determined criterion level that is assumed to result from

78 the subject learning the rules of the task; that is, which of the two shapes will lead to

79 reinforcement.

80 Using this basic paradigm, Harlow [1949] postulated that rhesus macaques (Macaca

81 mulatta) made more than a simple association between stimulus and reinforcement, suggesting

82 'cognitive mediation' occurred as subjects learned subsequent stimulus - reward associations

83 more quickly. This seemed to indicate they were extracting some level of information from their

84 first learning experience that they used to inform their actions in novel configurations. This has

85 since been termed 'learning to learn' or 'learning set formation' [Rumbaugh \& McCormack, 86 1967].

87 Since the early observations by Harlow, studies of learning set formation have shown that 88 many species including rhesus macaques (Macaca mulatta) and chimpanzees (Pan troglodytes),

89 can in fact 'learn to learn' with subsequent problems being solved in fewer presentations

90 [Passingham, 1981; Fobes \& King 1982]. However, gibbons have typically struggled with these

91 tests that require the subjects to solve different sets of similar problems over time and do not 
92 show the learning to learn pattern found in other species [Rumbaugh \& McCormack, 1967;

93 Tomasello \& Call, 1997; Abordo, 1976].

94 In the Rumbaugh and McCormack study [1967] five immature gibbons $(H$. lar $\mathrm{N}=2, H$.

95 moloch $\mathrm{N}=1 ; H$. pileatus $\mathrm{N}=2$ ) were presented with a simple discrimination task that required

96 subjects to push bins marked by a red square (reinforced) or a red circle (unreinforced) to reveal

97 a reward beneath the reinforced shape. The gibbons had a tendency not to push the bins at all -

98 taking upwards of 100 trials to acquire this basic motor action when compared to other primates

99 tested. However, once they were reliably making a choice, the gibbons did not appear to find

100 learning the discrimination any more difficult than the other apes and monkeys tested (Pongo,

101 Gorilla, Pan Sp., and Macacca) [Rumbaugh \& McCormack, 1967]. Unlike the other monkeys

102 and apes, when novel shape pairs were presented, gibbons seemed to learn each new association

103 as a completely new problem, taking the same number of trials to reach criterion performance as

104 they had for the first stimulus pair. Other species took fewer trials to learn each new association

105 suggesting they were using information gained during their first learning experience to inform

106 their choices on subsequent novel presentations. This seemed to be beyond the capacities of the 107 gibbons tested.

108 Other studies have provided contrasting results; for example, in a learning reversal study

109 (where the rewarded shape in a paired association task is 'reversed' once the subject reaches

110 criterion performance), Gosette [1970] reported gibbons (H.lar) did show evidence of learning to

111 learn making fewer errors than squirrel monkeys (Saimiri sp.) and owl monkeys (Aotus sp.), but

112 being inferior to capuchins (Cebus sp.). As some prior studies have found gibbons generally

113 struggle with learning set formations [Rumbaugh \& McCormack, 1967] while others have shown

114 conflicting evidence with better performance [Gosette, 1970], we were interested in clarifying 
D’Agostino and Cunningham 6

115 whether gibbons can use knowledge gained during one learning task to facilitate performance on

116 a similar but novel problem that would be a precursor to learning set formation.

117 Gibbons are large-brained primates with their relative brain size being comparable to the

118 great apes and large-brained monkeys [Cunningham \& Mootnick, 2009]; therefore, their reported

119 poor performance on discrimination tasks and little evidence of learning set formation is

120 surprising. Several gibbon behavioral characteristics could explain their apparent lack of

121 abilities and contradicting results of previous studies. Gibbons have notoriously short attention

122 spans [Fedor et al., 2008] potentially losing interest after only a few trials, impeding learning set

123 formation. Also, gibbons rely heavily on their visual cognitive abilities - it has been found that

124 gibbons are competent in detecting the visual orientation of other species as well as their own

125 and possess some knowledge of how visual gaze direction relates to external stimuli [Horton \&

126 Caldwell, 2006]. Therefore, results will be inconsistent if a subject with a short attention span

127 becomes distracted by a visual cue outside of the experiment.

128 The aim of this study was to determine whether gibbons could learn to associate preferred

129 foods with colored signals when the food items were hidden out of direct sight and once learned,

130 whether they could use their acquired learning to facilitate future learning. That is, once they had

131 learned a particular stimulus-reward association, would they learn subsequent novel associations

132 more easily? We also tested whether genera or sex had an impact on this ability based on

133 previous work that indicated differences in cognitive performance of gibbons were related to

134 subject-variables in that hoolock (Hoolock leuconedys) and siamang (Symphalangus syndactylus)

135 learned faster than the lar group (Hylobates sp.) [Cunningham et al., 2011] potentially due to

136 selection for flexibility caused by environmental variability.

\section{METHODS FOR EXPERIMENT 1}


D’Agostino and Cunningham 7

138

139

140

141

142

143

144

145

146

147

\section{Study site and subjects for Experiment 1 and 2}

149

150

151

152

153 Experiment 1 consisted of 13 gibbons (age 4-14 years) with representatives from three of the

154 four extant genera [Groves, 2002] (Hylobates pileatus $(N=2)$, Hylobates moloch $(N=2)$,

155 Symphalangus syndactylus $(N=2)$, Hoolock leuconedys $(N=7))$ (Table I). Ten gibbons

156 participated in Experiment 2 (age 4-23 years), five that were also subjects in Experiment 1 plus

157 two additional females $(H$. moloch $(N=1)$; H. pileatus $(N=1))$ and one male $(H$. pileatus $)$ that

158 took part in Experiment 2 only (Table II). Unavoidable problems prevented the same gibbons

159 being tested in both experiments; Violet (H. pileatus) was in isolation while recovering from an

160 arm injury and a family of $H$. leuconedys moved enclosures during testing. 
D’Agostino and Cunningham 8

161 Gibbons were housed in outdoor enclosures (10 X 3 X 4 m) with a connected section (4

$162 \mathrm{X} 3 \mathrm{X} 2.5 \mathrm{~m}$ ) generally available at all times that could be closed off to separate subjects as

163 required. This did not have a significant impact on the experiments as the gibbons were typically

164 separated a few times per week when the staff cleaned the enclosures and the veterinarian

165 checked the health of the animals. If we did notice any change in the animal's behavior that

166 could affect their performance in testing (due to separation or other distraction), we stopped the

167 current trial and resumed the following day. All enclosures were spaced by a minimum of $5 \mathrm{~m}$

168 and visual barriers and planted vegetation obstructed direct views between adjacent cages [see

169 Mootnick, 1997 for more details of enclosure design]. The gibbons were fed their normal daily

170 diet being fed five times per day with fruits and monkey biscuits in the morning and apples,

171 bananas and greens in the afternoon. The design of the enclosures allowed the gibbons to reach

172 their arms through fencing to access the feeding platforms used in the experiments (Figure 1).

173 Experiment 1

174 Experiment 1 consisted of two phases; Phase 1 was to determine the rank order of

175 preferred food items (banana, cucumber and monkey biscuits) for each individual. Phase 2

176 assessed whether they could learn an association between their preferred foods and a color cue.

177 In this discrimination task, the incentive was out-of-sight (concealed under a colored bucket) and

178 we assessed how quickly the gibbons could learn to associate the position of the food reward

179 with the cue. Three color/preferred food associations were presented consecutively, and we

180 evaluated whether subsequent configurations were learned more efficiently suggesting gibbons

181 were able to extract information from the first configuration to facilitate future learning. As prior

182 studies have showed gibbons struggle with learning set formations, possibly due to short

183 attention spans or being easily distracted, we attempted to limit these potential problems by 
184 focusing the gibbon's attention on visual cues within the experiment. A video that illustrates the 185 animals' behavior during both phases of Experiment 1 can be viewed by clicking the following 186 link: (AJP Production Team - Please Create Link to Video 1)

187 Phase 1 apparatus and procedure

188 Before testing began, subjects were desensitized to the new feeding platform that would 189 be used for the experimental trials. The subject's motor skills and grasping abilities were taken 190 into consideration when constructing the feeding platform, $130 \mathrm{~cm}$ long and $31 \mathrm{~cm}$ wide. If the 191 platform were smaller, the gibbons would be able to easily pick it up, flip it over, and potentially

192 lose interest in the test. If the platform were larger, it would make it difficult to transport to 193 various enclosures. Three plastic buckets, $25 \mathrm{~cm}$ tall and $15 \mathrm{~cm}$ in diameter were painted red, 194 yellow, and green. Three hinges were drilled into the wooden feeding platform $25 \mathrm{~cm}$ apart that 195 attached the buckets open-end down, to the feeding platform. The hinges allowed the buckets to 196 be flipped open by the gibbons to reveal food items hidden beneath (Figure 1).

197 To make the association between food and the feeding platform, the experimenter placed 198 a known favorite snack (as suggested by the staff at the GCC) of pumpkin seeds and blueberries 199 around and underneath the closed buckets on the feeding platform. This was to motivate the 200 gibbons to touch the buckets and feel comfortable with the task of opening them to reveal the 201 hidden food items. The experimenter presented the feeding platform to the subjects by sliding 202 along the floor until it was aligned with the enclosure and the rewards and buckets were within 203 the gibbons' reach when they extended their arm through the wire mesh. The pumpkin seeds 204 were scattered around the buckets and the buckets were closed over the blueberries. This feeding 205 platform set-up was same on every presentation in the desensitization phase. The platform was in 206 this position until all of the food items were consumed or for a maximum of five minutes if only 
207 a portion of the food was eaten. If the subject did not approach the platform at all, it was

208 presented again later that same day with a gap of two hours between desensitization trials. The

209 desensitization period continued on consecutive days until the subjects were comfortable using

210 the feeding platform. The gibbons were assumed to associate presence of the platform with a

211 reward when they readily approached, investigated and ate the pumpkin seeds, and finally

212 flipped the colored buckets and ate the blueberries. On average, five presentations of the feeding

213 platform were needed before there was evidence of an associative connection between the

214 feeding platform and the potential for reward.

215 Gibbons at GCC demonstrated preferences for specific food types when a variety of

216 choices were presented. This was recognized when staff at the center brought multiple items to

217 the enclosures and gibbons consistently picked and ate food in the same order. However, the

218 order of preference was not the same for every gibbon, although regardless of order, they were

219 usually individually consistent. As the experimental test relied on the association between

220 preferred food and colored signal being learned and remembered, we first had to assess

221 individual preference for the three items that would be used in the experimental tests.

222 General observations indicated that favorite foods were high in sugar and bright in color

223 (bananas, yams, and bell peppers). The least favorite foods had lower sugar content and were

224 usually plain in appearance (monkey biscuits). For these reasons, we predicted a favorite food

225 item would be banana, a moderate food item would be cucumber and the least favorite food item

226 would be monkey biscuits.

227 Each trial consisted of the experimenter presenting the feeding platform, within reaching

228 distance of each enclosure with the food items in full view. Incentives were placed on the

229 feeding platform out of view of the subjects in one of three locations that corresponded to the 
230 placement of the buckets that would be used on the test. Thus, rewards were in a horizontal line

231 relative to the enclosure fence, with the middle location being at the center of the feeding

232 platform and one reward positioned equidistant to the left and right of center. The position of the

233 three items changed in every trial in a randomly determined order. For example, on the first trial,

234 the piece of banana was on the left, cucumber in the middle and monkey biscuit on the right. For

235 the second, the position was switched so that the banana became central, cucumber right and

236 monkey biscuit left. The position of presentation was changed on every trial thereafter within the

237 constraint that the food items never appeared in the same position twice, to make sure the

238 gibbons did not get used to reaching for a particular food item in a specific direction. Notes were

239 taken of the order in which gibbons selected and ate the food items with the assumption that the

240 preferred food would be consumed first and the least preferred item, last.

241 Phase 2 - Associative learning of color cue and preferred reward

242 The purpose of Phase 2 was to determine if gibbons could associate colors to their rank

243 order of food items when they are out of direct sight (hidden under buckets). If the subjects were

244 able to make an association between the food items and color that signaled their preference

245 determined in Phase 1, they should select their preferred incentive as their first choice. All

246 buckets were baited with one item of the subjects preferred food (banana for all gibbons) and one

247 item of each of the other foods (cucumber and monkey biscuits) used in Phase 1 placed so that

248 when buckets were closed, the reward was out-of sight.

249 Studies using similar paradigms usually bait one location meaning the animal will only

250 receive a reward if they pick the correct cue. We chose to place a food reward under all buckets

251 and vary the 'value' of the food items to the individual (preferred or non-preferred) for a number

252 of reasons: 1) Sham baiting is normally employed of the non-rewarded locations to ensure the 
253 animals do not pick up on inadvertent experimenter-given cues during the baiting procedure

254 [Garber \& Dolins, 1996]. Rather than use a sham manoeuver which may still be different in 255 some way to an actual baiting, we baited all buckets ensuring our actions were the same at each

256 location; even though the actual placement of the rewards took place out of the subjects direct

257 sight, they could potentially have monitored the experimenter's movements. 2) By including the

258 two-distractor food items, we potentially increased the cognitive load by requiring subjects to

259 target their preferred food from three possible food/color cue associations presented in each trial.

260 3) As gibbons would be rewarded on all trials, their motivation to participate should not be

261 negatively affected across repeated trials.

262 Once baited, the apparatus were presented in the same way as for the food preference

263 trials with the feeding platform supporting the buckets being slid along at floor level until

264 aligned with the enclosure. The subjects could then extend their arms through the mesh and make

265 their choice of which bucket to flip open. The first trials allowed gibbons to learn the association

266 between the color cue and their preferred food item. The three colored buckets were baited out of

267 direct sight of the gibbons and the apparatus presented with the three food items concealed under

268 each one. On the first few presentations, the choice made would be inevitably random as the

269 gibbons did not know where their favorite food item was hidden. However, with repeated trials,

270 it was possible for the apes to acquire all the necessary knowledge to learn the association

271 between the color cue and the value of the reward beneath it. If they learned this association,

272 they should selectively choose the high value (preferred) food as their first choice on subsequent

273 trials. For example, during the first trial block, if 'green' was designated as the color cue that

274 would yield the highest reward, the preferred food item for the test subject (identified during

275 Phase 1) would be concealed under the green bucket for all trials in that block a block consists of 
276 all trials needed to reach criterion before moving to a new color association to a maximum of

277 100). For the first trials, the position of the green bucket was held constant to allow the

278 association between 'green' and the high value reward to be learned. Once the gibbon had

279 selected the green bucket as their first choice, the position of the green cue on all remaining trials

280 in the block was randomized; that is, the high value reward was always under the green bucket,

281 however, the position of the green cue varied on each trial.

282 The subject was considered to have learned the color-reward association when they

283 reached criterion performance set at 10 consecutive choices (out of a maximum100 trials) of the

284 preferred food type. Once criterion had been reached, presentations continued of the same color-

285 reward association until all 100 trials had been given. Trials that occurred after criterion had been 286 reached were referred to as 'post-criterion' trials.

287 Once gibbons had completed the required trials in the first block (learning and post-

288 criterion $\mathrm{N}=100$ ) with the first color/reward pairing, they moved to the second block of testing

289 where a new color/reward association had to be learned. Thus, individuals had to suppress the

290 previously learned rule and make a new association between a colored signal and reward. Three

291 blocks were presented (for each of the three color-reward associations to be learned) with each

292 block consisting of 100 presentations (learning and post-criterion trials) in total. The order of

293 presentation of each color-reward pairing was consistent across subjects. The trial was only

294 scored if the subject showed direct interest, measured by approaching the feeding platform

295 within 30 seconds. Trials were considered 'correct' if the gibbon chose the preferred item as their

296 first choice, however, they were allowed to continue lifting buckets until all rewards were

297 obtained (to maximize their motivation to take part). The trial was complete once all food items

298 has been consumed or after one minute elapsed. Any additional activity after one minute was 
299 considered "play" and the feeding platform was then taken away from the subject. If no choice

300 was made within the minute of exposure time, the trial was marked as incomplete and would be

301 resumed on the next day of testing. Similarly, if the subject lost interest during the trials, the

302 experiment was stopped and resumed at a later date, continuing where the subject left off until

303100 presentations were completed. The inter-trial duration was approximately three minutes.

304 This was the time needed to change the location of the buckets into a random order and bait the

305 buckets with the food items. Most subjects needed four sessions to complete 100 successful trial

306 presentations; however Reg and Truman (Hylobates sp.) needed five sessions.

307 Data analysis

308 Data were not normally distributed and so non-parametric statistics were used to evaluate

309 the effect of color on learning speed. Friedman's tests assessed differences in number of trials

310 needed to reach criterion performance (10 consecutive choices of preferred food first) for each

311 'color-preferred food' association (3 levels - Yellow, Green and Red). An extension of

312 generalized linear models (GZLM), generalized estimating equations (GEE) that accommodates

313 small, correlated within-subjects data sets and allows for comparisons between subjects [Garson

314 2013], determined how subject parameters (sex and genus) influenced learning the color-food

315 association. As data comprised counts of trials, Poisson loglinear regression with a log link

316 function was specified for all models with number of trials to criterion (as the response variable).

317 Genus, sex and food-color association (consecutive choices of preferred food first) were factors

318 in the model, with main effects tested. Hylobates was always the referent category for GEE

319 parameter estimates.

$320 \quad$ For the post-criterion performance analyses (trials that occurred after the initial

321 association had been learned), the number of trials varied across individuals due to differences in 
322 the acquisition of criterion performance and the maximum of 100 trials per individual summed

323 across learning and post-criterion trials (Post-criterion trial number $=$ Maximum number of trials

324 (100) - number of trials to criterion). Counts were therefore transformed into percentages for the

325 main analyses. Friedman's tests assessed differences in number of trials where preferred food

326 was selected first once learning had taken place for each color/preferred food association (3

327 levels - Yellow, Green and Red). GEE was again used to assess the retention of the color-reward 328 association across trials in the post-criterion stage with the same factors and covariates as

329 previously described; however, as data were no longer counts, a linear model was specified with

330 an identity link function. Again, Hylobates was the reference genus for the parameter estimates.

331 An additional Wilcoxon analysis was performed comparing the first ten trials post-criterion to

332 the last ten to evaluate whether performance was consistent throughout trials presented. Alpha

333 was set at 0.05 throughout and all tests were 2-tailed.

\section{RESULTS FOR EXPERIMENT 1}

335 Learning trials to criterion

336 All subjects tested demonstrated a preference for banana in the initial food choice trials

337 with nine of the 13 subjects choosing this food item first on $100 \%$ of presentations. The

338 remaining four subjects selected the banana first on the majority of preference trials in Phase 1

$339(86.67-93.33 \%)$ with cucumber being the occasional first choice. No subject selected monkey

340 biscuits as first choice in the preference tests. Therefore, banana was selected as the primary

341 color/reward referent for all subjects and responses scored as correct when the first choice made

342 on test trials was to select the location of this food type.

343 The number of trials needed to learn the primary food/reward association varied across

344 individuals (range 16-100); however, all subjects were able to reach criterion performance in all 
345 conditions within the 100-trial maximum. The color paired with the preferred food did not

346 influence learning speed across all individuals (Median (Yellow) = 26.00(IQR = 15.5), Median

$347($ Green $)=25.00(13.50)$, Median $($ Red $)=28.00(18.00)$, Freidman's test: $\chi^{2}(2)=0.840, p=$

348 0.657). However, there were significant differences between the genera on acquisition of the

349 color/reward association.

350 In general, Hylobates gibbons needed more presentations to learn the 'color-preferred

351 food' association than either Hoolock or Symphalangus (Table III). There was a significant effect

352 of genus on learning speed with Hoolock and Symphalangus needing significantly fewer trials

353 than Hylobates to learn the color associations (GEE: Hoolock B $=-0.677, \chi^{2}(1)=124.87, \mathrm{p}<$

354 0.001; Symphalangus B $\left.=-0.854, \chi^{2}(1)=33.94, \mathrm{p}<0.001\right)$ (Figure 3a). There were no

355 significant differences between sexes on number of trials to criterion $\left(\mathrm{GEE}: \mathrm{B}=-0.216, \chi^{2}(1)=\right.$

$3562.854, \mathrm{p}=0.091)$. Color paired with the preferred reward remained insignificant in this analysis

$357 \quad\left(\mathrm{GEE}: \chi^{2}(2)=1.965, \mathrm{p}=0.374\right)$.

358 Post-criterion trials

359 One subject did reach criterion in the all color-reward associations, however, only on the

360 final presentation of the green-reward configuration. This individual was therefore removed from

361 the post-criterion performance analyses. Selection of preferred food item was generally high

362 across all post-criterion trials $($ Mean $($ Yellow $)=91.56(\mathrm{SE}=1.35) \%$, Mean $($ Green $)=$

$36378.71(7.39) \%$, Mean $($ Red $)=88.51(1.88) \%$, , with no significant differences in performance

364 across color cues (GEE: $\left.\chi^{2}(1)=1.941, p=0.379\right)$. Sex was also non-significant as a predictor of

365 post-criterion performance $\left(\mathrm{GEE}: \chi^{2}(1)=0.001, \mathrm{p}=0.970\right)$. Genus however, did significantly

366 impact performance with Symphalangus performing significantly better than Hylobates (B

$\left.367=10.184, \chi^{2}(1)=10.468, \mathrm{p}<0.01\right)$. Hoolock also obtained fewer rewards than Symphalangus, 
368 although performance was not significantly different from that of Hylobates subjects $(\mathrm{B}=4.961$,

$\left.369 \chi^{2}(1)=1.838, p=0.175\right)$ (Figure 3b). No color/preferred food association showed diminished

370 persistence in the first block post-criterion compared to the last block (Wilcoxon matched pairs:

371 Yellow $\mathrm{Z}=0.00, \mathrm{p}=1.00 ;$ Green $\mathrm{Z}=-0.905, \mathrm{p}=0.366$; Red $\mathrm{Z}=-0.707, \mathrm{p}=0.480$ ).

\section{DISCUSSION FOR EXPERIMENT 1}

373 We were able to determine the rank order of food items and the most preferred for all

374 subjects was banana. We also determined that gibbons could successfully select their preferred

375 food when colored buckets covered it, suggesting they could use the color as a signal to the high

376 value food's location. There were no differences across color-reward associations, indicating

377 learning was not affected by the particular color/reward.

378 There was no evidence the gibbons tested here generalized from their learning in the first

379 round to other rounds as there were no differences between number of trials to reach criterion

380 performance with each successive color-reward association to be learned. Effectively, they

381 seemed to be learning each new association from scratch - as if they had not seen or experienced

382 the task before. This could be the result of multiple food items being used that distracted the

383 gibbons and obscured the intended question of whether gibbons can generalize and use

384 information learned in the first association to inform their learning in subsequent novel

385 associations.

\section{INTRODUCTION FOR EXPERIMENT 2}

387 Since we found the gibbons tested showed no evidence of generalizing from their

388 learning, we changed the methods and feeding platform in a second experiment where only one

389 food item would be searched for with three possible locations cued by a colored background that

390 we hoped would provide a more salient cue to the rewards location. We aimed to further 
391 investigate the flexibility of learning by assessing whether there was any evidence of gibbons

392 generalizing from the first color/reward association learned to other, novel associations.

393 In Experiment 1, gibbons were required to learn an association between a preferred food

394 and specific color while simultaneously presented with two further possible color choices that

395 would also provide a reward, all be it of lower value. In Experiment 2, we reduced the cognitive

396 load be removing the secondary food items and the competing color cues in an attempt to focus

397 the gibbon's attention on a single preferred food reward (banana). We hypothesized that by

398 simplifying the cues presented, the gibbons tested may be better able to extract information from

399 their initial learning experience that would aid them in subsequent novel associations: that is,

400 they should be able to generalize from their learning in the first presentations and show improved

401 performance in subsequent similar learning situations.

\section{METHODS FOR EXPERIMENT 2}

403 The feeding platform for Experiment 2 required the gibbons to reach through doors to

404 select a hidden food item. Only one door was baited with a piece of banana and the position was

405 determined by the color cue on the front of the platform that measured 1.2 meters long, 0.3

406 meters wide and $0.6 \mathrm{~m}$ high. It was large enough for the experimenter to hide behind and "reload"

407 the food item between trials without subjects seeing where the food item was placed. The

408 experimenter also wore dark sunglasses during the trials to avoid giving inadvertent gaze cues to 409 the subject.

410 The front of the feeding platform had three doors located at the base - on the left, center

411 and right. The doors were six inches long and six inches wide - large enough for the animals to

412 easily reach through (Figure 2). On the back of the doors was secured a sheet of heavy fabric that

413 prevented the animals seeing through to the reward. Thus, in order to retrieve a reward, the 
414 subject had to move aside the fabric and reach through the door. Wooden dividers acted as

415 barriers on the offside of the feeding platform, between the three possible feeding locations, to

416 prevent the animals from reaching in one door and stretching their arms to the location of a food

417 item behind another door. Three laminated color boards were inserted onto the front of the

418 feeding platform behind a Plexiglas screen. The reason for the Plexiglas was because it is easily

419 cleaned between trials and provided a mechanism to hold the interchangeable laminates in place

420 (Figure 2). The laminates provided the color cue and varied dependent on the trial block between

421 red, blue and yellow.

422 A video that illustrates the animals' behavior as well as visually displaying the

423 experimental design of Experiment 2 can be viewed by clicking the following link: (AJP

424 Production Team - Please Create Link to Video 2)

425 Desensitization and Learning Trials

426 Similar to Experiment 1, desensitization trials were conducted before experimental trials

427 began. This was to desensitize the subjects to using the new feeding platform. The experimenter

428 placed pumpkin seeds in front of the closed doors and blueberries behind the closed doors. The

429 feeding platform was then presented to the subjects by positioning it outside the enclosure,

430 within direct reach of the gibbons. This feeding platform set-up was exactly the same on every

431 presentation.

432 Identical to Experiment 1, the platform was in this position until all of the food items

433 were consumed or for a maximum of five minutes if only a portion of the food was eaten. The

434 gibbons were assumed to have learned to associate the presence of the platform with a reward

435 when they readily approached, investigated and ate the food items. If the subject did not

436 approach the platform at all, the presenter would try again later that same day with a gap of two 
437 hours between desensitization trials. The desensitization period continued on consecutive days

438 until the subjects were comfortable using the feeding platform.

439 The gibbons were recorded as associating the presence of the platform with a reward

440 when they completed two steps: 1) readily approached, investigated and ate the pumpkin seeds

441 and 2) reached through the doors and ate the blueberries. It took slightly longer for the gibbons in

442 Experiment 2 to make this association with as many as seven presentations of the feeding

443 platform required before there was evidence of an associative connection between the feeding

444 platform and the potential for reward in all gibbons. Once all subjects were consistently using the

445 new platform, we began trials to provide an opportunity for the gibbons to learn the task

446 requirements for Experiment 2.

447 During the initial learning trials, a food reward (piece of banana) was concealed behind

448 one of the doors which the gibbon was given the opportunity to retrieve. The location of the food

449 reward was determined by the color background (laminate) that was held constant to allow

450 gibbons to make the association between the rewards position and the color cue. Each subject

451 was initially presented with the red background which signified the reward (banana) was located

452 behind the left door. This configuration was repeatedly presented until the gibbon reliably chose

453 the left door, and retrieved the banana on their first selection for three consecutive trials

454 (criterion for the learning phase). Once criterion was reached for this color/position association,

455 the background color and the position of the reward were changed to a blue background with the

456 banana behind the center door, and finally a yellow background with the banana behind the right

457 door (Table II) with criterion being three consecutive correct choices for each color/reward

458 association. 
459 Similar to Experiment 1, a trial was only scored if the subject showed direct interest, 460 measured by approaching the feeding platform within 30 seconds. The trial was marked as 461 correct if the subjects' first choice was for the rewarded location and a trial was considered 462 complete once the food item had been consumed or after one minute elapsed (if no choice was 463 made). Any additional activity after one minute was considered "play" and the feeding platform 464 was then taken away from the subject. If the food item was not consumed within the minute of 465 exposure time, the trial was marked as incomplete and would be resumed on the next day of 466 testing. Similarly, if the subject lost interest during the trials, the experiment was stopped and

467 resumed at a later date, continuing where the subject left off until all possible presentations were 468 completed. The inter-trial duration for all trials (learning and experimental) was approximately 469 three minutes.

470 Once criterion was reached for all three possible positions in block 1 (all trials where 471 position of reward was always placed in accordance with the color/reward associations learned in 472 the training trials), the subject moved on to the experimental trials (see below). On completion of 473 the first round of testing in block 1 (learning and experimental trials), the learning trials for block 4742 commenced following the same protocol as for block 1, where the position of the reward 475 changed dependent on the color background association (block 2 - Red/Center, Blue/Right, 476 Yellow/Left). Thus, the subject experienced a series of learning trials followed by the 477 experimental trials for block 1 , and then returned to learning trials for block 2 . Once the 478 experimental trials for block 2 were completed, the learning trials for block 3 commenced (Block $4793-$ Red/Right, Blue/Left, Yellow/Center).

480 Experimental Trials 
D’Agostino and Cunningham 22

481

482

483

484

485

486

487

488

489

490

491

493

494 learning trials.

495 Data analysis

496 Trials were scored as correct if the gibbon retrieved the food reward with their first

497 choice when presented with the apparatus. For the learning trials, the number of trials needed to

498 reach criterion (three consecutive correct choices) were analyzed using Friedman's repeated

499 measures test to assess whether any background color association was more difficult to learn.

500 GEE models were used to evaluate whether performance on the learning trials differed across

501 genera or by sex. As data were counts, Poisson distribution with a log function was specified,

502 genus and sex being factors in the model. 
Performance on the experimental trials was evaluated at the group and individual level.

504 To determine the effects of learning on subsequent color/position associations, a GEE was

505 conducted with a binomial distribution and logit link function. Events in trials data were

506 specified with a fixed value of 30 (number of experimental trials per block). Genus and sex were

507 added as factors in the model with Hylobates as the referent category for the former.

508 Whether the gibbons were using the color cues to guide their choices was assessed by

509 determining whether they were selectively choosing the correct door significantly above chance.

510 Binomial tests with the test proportion set at 0.33 (as there were three possible choices) were

511 conducted at the group and individual level. During the observations, a location bias seemed

512 possible. Therefore, Chi square goodness of fit tests were used to evaluate whether the gibbons

513 as a group and individually, were selecting any particular door (center, left or right) more that

514 would be expected. Alpha for all tests was set at 0.05 .

\section{RESULTS FOR EXPERIMENT 2}

\section{$516 \quad$ Learning trials}

517 Eight gibbons cooperated enough to engage in experimental trials and generate useable

518 data. Although it was planned to include representatives from all four genera, none of the

519 northern white-cheeked gibbons (Nomascus leucogenys) would participate in the experiment.

520 Only one female white-cheeked gibbon would approach the feeding platform and remove the

521 food items in the training trials. However, this gibbon was apprehensive to reach through the

522 doors to remove the hidden food items in the learning or experimental trials. The male white-

523 cheeked gibbons would not approach the feeding platform. This could be due to the daily feeding

524 routine that involves feeding each gibbon separately with some food being handed directly or

525 gently tossed to them to catch. They do not typically approach a feeding platform, hesitated 
526 doing so in these experiments, which made them unwilling participants. Therefore, the final

527 sample consists of subjects from three genera (Table I).

528 The number of trials needed to learn each background color/reward association ranged

529 from 3 to 11 with most individuals reaching criterion in 5 or less trials for each color-reward

530 configuration. There were no significant differences between number of trials to criterion for

531 each background color association in the learning trials (Median (Yellow background) $=$

532 14.50(IQR = 6.0), Median $($ Blue background $)=13.00(9.0)$, Median $($ Red background $)=$

$53315.00(9.00)$, Freidman's test: $\left.\chi^{2}(2)=2.769, p=0.250\right)$. There was a main effect of genus on

534 number of trials to criterion with Hoolock and Symphalangus needing fewer presentations than

535 Hylobates (GEE: Hoolock B $=-0.529, \chi^{2}(1)=99.887, \mathrm{p}<0.001 ;$ Symphalangus $\mathrm{B}=-0.493$,

$536 \chi^{2}(1)=31.124, p<0.001$ ) (Figure 4). Sex was not a significant predictor of performance in the

537 learning trials (GEE: $\left.\chi^{2}(1)=2.971, \mathrm{p}=0.085\right)$.

538 Experimental trials

539 Consistent with the learning trials, there was no evidence that the gibbons were

540 generalizing from the learned association in block 1 to the novel associations in block 2 and 3

541 (GEE: $\left.\chi^{2}(2)=0.719, p=0.698\right)$. The effect of genus on performance in the experimental trials

542 was significant with Symphalangus gaining significantly more rewards than Hylobates

543 (Symphalangus $\operatorname{Mdn}=57.00$, Hylobates $\operatorname{Mdn}=43, \mathrm{~B}=0.658, \chi^{2}(1)=65.161, \mathrm{p}<0.001$ ).

544 Hoolock did gain more rewards than Hylobates, however this did not reach significance

545 (Hoolock $\left.\mathrm{Mdn}=46, \mathrm{~B}=0.290, \chi^{2}(1)=2.771, \mathrm{p}=0.096\right)$. Sex was again non-significant in this 546 analysis $\left(\chi^{2}(1)=0.791, p=0.372\right.$. 
547 As a group, gibbons were selectively choosing the correct reward location above chance

548 levels gaining 378 out of 720 possible rewards collectively (Binomial: $p<0.001$ ). Individual

549 performance is shown in Table IV, with six gibbons selecting the rewarded door significantly

550 above chance overall. Within this group, three gibbons dropped below chance level on one set of

551 the three with the remaining three performing above chance in all sets. Developing a bias for one

552 location over the others may have influenced performance. Five of the eight gibbons showed a

553 positional bias (Table V) selectively favoring the center door in general. This bias did not

554 however emerge consistently across all blocks.

\section{GENERAL DISCUSSION}

556 In Experiment 1, we found no evidence the gibbons tested generalized from their learning 557 and we cautioned this could be the result of multiple food items and competing color cues being 558 used that distracted the gibbons. Therefore, we changed the experimental design to a more

559 specific task in Experiment 2 to focus the gibbon's attention on a single food/color association to 560 see if the gibbons were able to use information from their first learning experience of the task to

561 facilitate future learning of novel color/reward associations. Despite making these changes, the 562 data show they learned each new association from scratch - as if they had not seen or

563 experienced the task before as in Experiment 1. Our findings are similar to other studies

564 [Rumbaugh \& McCormack, 1967] that found gibbons struggle with learning set formation and 565 do not show the learning to learn pattern as found in other species. However, once the learning 566 association was made, the gibbons performed well above chance. We found no differences

567 across color associations indicating learning was not affected by the particular color / reward 568 association 
569 The results reported here could be due to the small sample size and because of the captive

570 environment in which these gibbons were raised. Karenina and Marlow, siamang gibbons

571 (Symphalangus syndactylus) who performed quite well in the tasks reported here, had a lot of

572 human interaction from a young age. Perek, a Javan gibbon (Hylobates moloch) who did not

573 perform as well in these experiments, was born at Howlets Wild Animal Park, UK, and did not

574 have as much human interaction.

575 The Gibbon Conservation Center provided a unique opportunity being the only institution

576 in the world to house and breed all four genera of gibbon [Mootnick, 1997]. However, obtaining

577 large sample sizes is very challenging as there are very few gibbons (particularly of the Hoolock

578 genus) in captivity. Another possibility for these results is the low criterion level set for

579 Experiment 2. We lowered the number of consecutive correct learning trials to compensate for

580 the gibbons short attention spans but this may not have given them sufficient time to really learn

581 the association. Results from previous experiments where hundreds of trials have been presented

582 in the learning phase [Rumbaugh \& McCormack, 1967] suggest performance may have been

583 much better had they been given more opportunity to learn. Despite such a low criterion, many

584 of the gibbons did perform well above chance in the experimental trials although they may not

585 have had the opportunity to learn the rules of the task that could then be generalized to future

586 novel presentations.

587 We found a key difference in the learning and association rates between male and female

588 gibbons with the males learning at a faster rate than females. Reproductively active females may

589 be highly motivated to explore potential resources due to the additional energy requirements of

590 pregnancy and lactation; however, increased investment in developing offspring could make

591 them more guarded in their investigations and so reluctant to engage with unknown objects such 
592 as our testing equipment [Cunningham et al., 2011]. Males, without the investment in offspring,

593 may be more devoted to the search of potential resources and more willing to explore novel

594 objects.

595 We have shown that when given provisioned food items, the subjects tested have

596 preferred foods and can associate color signals with these foods. However, there were no

597 differences across color associations and the rate of learning was no different from the colors red,

598 green, yellow, blue, or if the food item was on the left, center or right. This suggests the subjects

599 can associate each of the colors tested to the food items at the same rate and accuracy with no

600 particular preference to a specific color or location.

601 The Hylobates sp. had the slowest learning rate before they made the association between

602 their preferred foods and the color cue in Experiment 1 and 2. Hoolock learned the association

603 significantly quicker, needing fewer trials in all color/preferred food association learning phases.

604 For all gibbons tested, males learned faster than the females, however age did not impact

605 learning rate although younger gibbons did show more inconsistencies in their performance,

606 selecting other food items as their first choice on some trials in Experiment 1, especially in the

607 post-criterion phase. This however did not have a significant impact on results with all subjects

608 selecting their preferred food item with a high level of accuracy in all post-criterion trials.

609 Possible Foraging Implications

610 This ability to associate colors to preferred foods can be an advantage in a changing

611 environment. For instance, Hoolock gibbons, which performed well compared to the lar-group in

612 this study, are from an environment that is variable and resources can be scarce. The lar-group

613 gibbons, who performed the poorest, live in an area where the climate is more stable and food is

614 likely to be plentiful year round [Brockman \& van Schaik CP, 2005; Curran \& Leighton, 2000]. 
615 This suggests that animals required to search more vigorously for high quality food items due to

616 environmental variability are more flexible in their learning than animals that do not have the

617 same foraging pressures.

618 There have been previous investigations that support the assumption that trichromatic 619 color vision provides an advantage related to foraging [Caine \& Mundy, 2000; Nagle \& Osorio, 620 1993; Osorio \& Vorobyev, 1996; Regan et al., 1998, 2001; Lucas et al., 1998]. However, these 621 investigations did not take into account using colors as visual landmarks or make the direct 622 connection between preferred foods and how animals can associate colors to these foods.

623 To consider possible foraging implications as well as the ecological validity of the 624 experimental design and results reported here, the fact that color is an ephemeral cue in the 625 environment must be taken into account. In general, forests retain a homogenous set of colors 626 with changes in accordance with fruit ripening and leave leaf maturation across time. Mostly, 627 animals focus on more permanent environmental cues while navigating such as the size and 628 shape of trees. However, if an animal can take advantage of the changing colors in the 629 environment and learn to make the association of these new color cues to the location of ripe 630 fruit, they will have a foraging advantage over animals that do not possess this ability.

631 Resource availability is rarely ideal. Research has found yearly fluctuations in the 632 availability of resources which has a significant impact on the demography, foraging ecology, 633 life history patterns, population density, anti-predator strategies, and social behaviour of 634 nonhuman primates [Brockman \& van Schaik, 2005]. When preferred foods are scarce, optimal 635 foraging theory predicts primates should attempt to maintain the net energy intake required for 636 physiological maintenance by either increasing their ranges to continue utilizing preferred foods 637 or relying on alternative foods [e.g., MacArthur \& Pianka, 1966; Stephens \& Krebs, 1986; van 
638 Schaik \& Brockman, 2005]. Thus, primates may act as "energy maximizers," increasing their

639 day journey length (DJL) or home range size to consume as many scarce preferred resources as

640 possible, or "energy minimizers," decreasing their DJL and home range size while consuming

641 more low-quality resources [e.g., Clutton-Brock, 1977; Boinski, 1987; Barton et al., 1993;

642 Barton, 1998, 1999; Overdorff, 1993; Hemingway \& Bynum, 2005; Riley, 2007].

643 Given a gibbon's body size, morphological and physiological adaptations, grouping

644 patterns and social organization, they fit the description of energy maximizers. They spend a

645 great deal of energy searching for and traveling between patches of foods. They use their

646 adaptations, expand their ranges, and continue to search for preferred foods that are usually high-

647 energy fruits that are bright in color. In order understand the foraging advantages conferred by

648 the ability to associate ephemeral color cues to preferred food items in relation to resource

649 distribution, further testing is needed in both captivity and nature.

650 Conclusions and Future Study

651 Since we have shown the gibbons tested had difficulty generalizing from their learning in

652 one context and using it to facilitate learning in a similar situation, we must conclude that these

653 apes are capable of making color/reward associations but cannot use their learning flexibly. This

654 makes it unlikely that they would, with further testing, demonstrate learning set formation

655 consistent with results from earlier research [Rumbaugh \& McCormack, 1967]. However, the

656 emergence of learning to learn patterns may occur with the use of more structured small steps to

657 facilitate learning of the rules of the task in an incremental way. For example, repeated

658 presentations of one color cue that signifies a reward presented with a neutrally colored

659 unreinforced cue before changing the color cue but not the rule (color would still indicate the

660 position of the reward) after the subject experienced many more trials than used here may allow 
661 gibbons to extract the general rule that can be used to solve future novel presentations rather than

662 just a simple stimulus-reward association. With this more intensive training regime, they may be

663 able to apply their learning in a more flexible way.

\section{ACKNOWLEDGEMENTS}

665 We would like to thank the Gibbon Conservation and the staff, especially Chris Roderick

666 and Gabriella Skollar who provided generous support and advice through the entire

667 investigation. We would also like to thank the late Alan Mootnick, the founder of The Gibbon

668 Conservation Center, for his lifelong hard work and dedication to gibbons. This project was

669 supported by a grant from the California State University, Los Angeles - office of graduate

670 studies and research's RSCA fund to support research scholarship and creative activity.

\section{REFERENCES}

672 Abordo EJ. 1976. The learning skills of gibbons. In: DM Rumbaugh editor. Gibbon and Siamang

673 Vol. 4: Behaviour, locomotion and other behaviours in captive gibbons; cognition.

$674 \quad$ Karger: Basel p. 106-134.

675 Barton RA. 1998. Visual specialization and brain evolution in primates. Proceedings of the

676 Royal Society of London B-265, 1933-1937.

677 Barton RA. 1999. The Evolutionary Ecology of the Primate Brain. In: Lee, PC editor, 678 Comparative primate socioecology. Cambridge: Cambridge University Press. p 167-203.

679 Barton RA, Whiten A, Byrne RW, English M. 1993. Chemical composition of baboon plant 680 foods: implications for the interpretation of intra- and inter-specific differences in diet. $681 \quad$ Folia Primatologica 61:1-20.

682 Brockman DK, van Schaik CP. 2005. Seasonality in primates: Studies of living and extinct 683 human and non-human primates. Cambridge: Cambridge University Press. 
684 Boinski S. 1987. Habitat use by squirrel monkeys in a tropical lowland forest. Folia 685 Primatologica 49:151-167.

686 Caine NG, Mundy NI. 2000. Demonstration of a foraging advantage for trichromatic marmosets 687 (Callithrix geoffroyi) dependent on food color. Journal of Biological Sciences 267:439688444.

689 Clutton-Brock TH. 1977. Some aspects of intraspecific variation in feeding and ranging behavior 690 in primate. In: T.H. Clutton-Brock (editor): Primate Ecology New York: Academic Press. 691 p. 539-556.

692 Cunningham CL, Anderson J, Mootnick AR. 2006. Object manipulation to obtain a food reward 693 in hoolock gibbons, Bunopithecus Hoolock. Animal Behavior. 71:621-629.

694 Cunningham CL, Anderson J, Mootnick AR. 2011. A sex difference in effect of prior experience 695 on object-mediated problem-solving in gibbons. Animal Cognition 14:599-605.

696 Cunningham CL, Mootnick AR. 2009. Gibbons. Current Biology 19:R543-R544. 697 doi:10.1016/j.cub.2009.05.013

698 Curran LM, Leighton M. 2000. Vertebrate responses to spatiotemporal variation in seed 699 production of mast fruiting dipterocarpaceae. Ecological Monographs 70:101-128.

700 Deegan JF, Jacobs GH. 2001. Spectral sensitivity of gibbons: implications for photopigments 701 and color vision. Folia Primatologica 72:26-29.

702 Fedor A, Szerencsy N, Skollar G, Ujhelyi M. 2008. Object permanence tests on gibbons 703 (Hylobatidae). Journal of Comparative Psychology 122:403-417.

704 Forbes JL, King JE. 1982. Measuring primate learning abilities. In: JL Forbes and JE King 705 editor. Primate Behavior. New York: Academic Press. p. 289-326.

706 Garber PA., Dolins FL. 1996. Testing Learning Paradigms In The Field: Evidence for Use of 
Spatial and Perceptual Information in Rule-Based Foraging in Wild Moustached Tamarins. In M. A. Norconk, A. L. Rosenberger, and P. A. Garber (Eds), Adaptive Radiations of Neotropical Primates. New York, NY: Plenum Press.

710 Garson GD. 2013. Generalized linear models and generalized estimating equations; statistical 711 associates blue book series. Statistical Associates Publishers.

712 Gossette RL. 1970. Comparisons of SDR performance of gibbons and three species of new world 713 monkeys on a spatial task. Psychonomic Science 19:301-303.

714 Groves C. 2002. Primate taxonomy. London: Smithsonian Institutional Press

715 Harlow HF. 1949 The formation of learning sets. Psychological Review 56:51-65.

716 Hemingway CA, Bynum N. 2005. The influence of seasonality on primate diet and ranging. In:

717 DK Brockman and CP van Schaik editor. Seasonality in primates: Studies of living and 718 extinct human and non-human primates. Cambridge: Cambridge University Press. p. 57$719 \quad 104$.

720 Horton KE, Caldwell C. 2006. Visual co-orientation and expectations about attentional 721 orientation in pileated gibbons (Hylobates pileatus). Behavioural Processes 72:65-73.

722 Jacobs GH. 1993. The distribution and nature of color vision among the mammals. Biological $723 \quad$ Reviews 68:413-471.

724 Jacobs GH, Neitz M, Deegan JF, Neitz J. 1996. Trichromatic color vision in New World 725 monkeys. Nature 382:156-158.

726 Lucas PW, Darvell BW, Lee PKD, Yuen TDB, Choong MF. 1998. Color cues for leaf food 727 selection by long-tailed macaques (Macaca fascicularis) with a new suggestion for the 728 evolution of trichromatic color vision. Folia Primatologica 69:139-152.

729 MacArthur RH, Pianka ER. 1966 On the optimal use of a patchy environment. The American 
Naturalist 100:603-609.

731 Mootnick AR. 1997. Management of gibbons Hylobates spp. at the international center for

732 gibbon studies, California: with a special note on Pileated gibbons Hylobates pileatus.

733 International Zoo Yearbook 35:271-279.

734 Nagle MG, Osorio D. 1993. The tuning of human photo pigments may minimize red-green

735 chromatic signals in natural conditions. Proceedings of the Royal Society of London B

$736 \quad 252: 209-213$.

737 Osorio D, Vorobyev M. 1996. Color vision as an adaptation to frugivory in primates.

738 Proceedings of the Royal Society of London B 263:593-599.

739 Overdorff DJ. 1993. Similarities, differences, and seasonal patterns in the diets of Eulemur

740 rubriventer and Eulemur fulvus rufus in the Ranomafana National Park, Madagascar.

741 International Journal of Primatology 14:721-754.

742 Passingham RE. 1981. Primate specialization in brain intelligence. Symposium of the Zoological $743 \quad$ Society of London 46:361-388.

744 Regan BC, Julliot C, Simmen B, Vienot F, Charles-Dominique P, Mollon JD. 1998. Frugivory

745 and color vision in Alouatta seniculus, a trichromatic platyrrhine monkey. Vision

$746 \quad$ Research 38: 3321-3327.

747 Regan BC, Julliot C, Simmen B, Vienot F, Charles-Dominique P, Mollon JD. 2001. Fruits, 748 foliage and the evolution of primate color vision. Philosophical Transactions of the Royal $749 \quad$ Society of London B 356:229-283.

750 Riley EP. 2007. Flexibility in diet and activity patterns of Macaca tonkeana in response to 751 anthropogenic habitat alteration. International Journal of Primatology 28:107-133.

752 Rumbaugh DM, McCormack C. 1967. The learning skills of primates; a comparative study of 
apes and monkeys. In: D Stark, R Schneider, HJ Ruhn editor. Progressive Primatology.

754 Hillsdale NJ; Lawerence Erlbaum Associates. p. 289-306.

755 Stephens DW, Krebs JR. 1986. Foraging theory. Princeton: Princeton University Press.

756 Tomasello M, Call J. 1997. Primate Cognition. Oxford University Press, New York.

757 Van Schaik CP, Brockman DK. 2005. Seasonality in primate ecology, reproduction and life

758 history: an overview. In: D.K. Brockman and C.P. van Schaik editor. Seasonality in

759 primates: studies of living and extinct human and non-human primates. Cambridge:

760 Cambridge University Press. p. 3-20.

761

762

763

764

765

766

767

768

769

770

771

772

773

774

775

776

777

778

779

780

781

782

783

784

785

786

787

788

789

790 
Figure Legends

Figure 1. Betty (Female H. leuconedys) engaged in Experiment 1. The gibbons reached through the fencing and flipped open the buckets to reveal the food items. The buckets were drilled in place with hinges that allowed them to be easily flipped and replaced to the different sets.

807

808

809

810

Figure 2. Feeding platform used for Experiment 2. This platform required the gibbons to reach through doors to select the hidden food item. Three laminated color boards were inserted onto the front of the feeding platform behind a Plexiglas screen.

811

812

813

814

815

816

817

818

819

820

821

822

823

824

825

826

827

828

829

830

831

832

833

834

835

836

Figure 3: Adjusted means for number of trials needed to reach criterion performance (10 consecutive first choices of preferred food type) by genus (a) and percentage of correct responses by genus in the post-criterion phase (b). Error bars represent \pm 1 SE. $* *$ denotes significance at $p<$ 0.001

Figure 4: Adjusted means for number of trials to reach criterion performance in the learning trials of Experiment 2 by genus. Error bars represent \pm 1 SE. ** denotes significance at the 0.001 level. 
D’Agostino and Cunningham 36

840

841

842

843

844

845

846

847

848

849

850

851

852

853

854

855

856

857

858

859

860

861

862
Tables:

Table I. Names, species, sex, and age of the subjects in Experiment 1 and Experiment 2.

\begin{tabular}{lllll}
\hline \multicolumn{1}{c}{ Subject } & \multicolumn{1}{c}{ Species } & \multicolumn{1}{c}{ Sex } & \multicolumn{1}{c}{ Age in yrs } & Experiment \\
\hline Marlow & Symphalangus syndactylus & Female & 8 & $1 \& 2$ \\
Karenina & Symphalangus syndactylus & Female & 14 & $1 \& 2$ \\
Khin Maung Win & Hoolock leuconedys & Male & 5 & $1 \& 2$ \\
U Myint Swe & Hoolock leuconedys & Male & 4 & $1 \& 2$ \\
Hmawe Ni & Hoolock leuconedys & Female & 11 & $1 \& 2$ \\
Betty & Hoolock leuconedys & Female & 14 & 1 \\
Chan Thar & Hoolock leuconedys & Female & 6 & 1 \\
U Maung Maung & Hoolock leuconedys & Male & 12 & 1 \\
Win Bo & Hoolock leuconedys & Male & 10 & 1 \\
Reg & Hylobates moloch & Male & 13 & 1 \\
Perak & Hylobates moloch & Male & 11 & 1 \\
Truman & Hylobates plieatus & Male & 10 & 1 \\
Violet & Hylobates pileatus & Female & 4 & 1 \\
Tuk & Hylobates pileatus & Female & 20 & 2 \\
Chloe & Hylobates moloch & Female & 23 & 2 \\
Domino & Hylobates pileatus & Male & 18 & 2 \\
\hline
\end{tabular}


2

3

4

5

8

9

10
863 Table II: Experimental sets used in Experiment 1 and 2.

864

\begin{tabular}{lcc}
\hline Sets & Experiment 1 & Experiment 2 \\
\hline 1 & Red left, yellow center, green right & Red left, blue center, yellow right \\
2 & Red left, green center, yellow right & Blue right, yellow left, red center \\
3 & Yellow left, red center, green right & Yellow center, red right, blue left \\
4 & Yellow left, green center, red right & \\
5 & Green left, red center, yellow right & \\
6 & Green left, yellow center, red right & \\
\hline
\end{tabular}

865

866

$867{ }^{1}$ For Experiment 1, color refers to the bucket covering the food and position (left, right, center) 868 designates the position of the colored bucket on the feeding platform.

$869{ }^{2}$ In Experiment 2, the color refers to the background and the position to the location of the food 870 reward. 
D’Agostino and Cunningham 38

902 Table III: Unadjusted means for number of trials to criterion performance.

903

\begin{tabular}{llll}
$\begin{array}{l}\text { Preferred } \\
\text { food color } \\
\text { association }\end{array}$ & $\begin{array}{l}\text { Hoolock }(\mathbf{n}=7) \\
\text { mean }(\text { SE) }\end{array}$ & $\begin{array}{l}\text { Hylobates }(\mathbf{n}=4) \\
\text { mean(SE) }\end{array}$ & $\begin{array}{l}\text { Symphalangus }(\mathbf{n}=2) \\
\text { mean(SE) }\end{array}$ \\
\hline YELLOW & $22.29(1.782)$ & $50.25(11.161)$ & $29.50(10.500)$ \\
GREEN & $28.57(2.277)$ & $48.50(19.294)$ & $23.00(0.000)$ \\
RED & $27.14(2.064)$ & $48.75(11.116)$ & $21.00(0.000)$ \\
\hline
\end{tabular}

904

905

${ }^{1}$ Ten consecutive correct choices for three genera of gibbon for each color-preferred food 906 association in Experiment 1

907

908

909

910

911

912

913

914

915

916

917

918

919

920

921

922

923

924

925

926

927

928

929

930

931

932

933

934

935

936

937

938

939

940

941 
2

942 Table IV: Number of correct responses in the experimental trials of Experiment 2. 943

\begin{tabular}{|c|c|c|c|c|c|c|c|c|}
\hline Subject & \multicolumn{2}{|c|}{ S1 } & \multicolumn{2}{|c|}{ S2 } & \multicolumn{2}{|c|}{ S3 } & \multicolumn{2}{|c|}{ Total } \\
\hline & Correct & $p$ & Correct & $p$ & Correct & $p$ & Correct & $p$ \\
\hline Marlow & 19 & $0.001 * * *$ & 19 & $0.001 * * *$ & 18 & $0.002 * *$ & 56 & $0.001 * * *$ \\
\hline $\begin{array}{l}\text { Khin } \\
\text { Maung Win }\end{array}$ & 15 & $0.001 * * *$ & 12 & 0.002 & 11 & 0.001 & 38 & 0.042 \\
\hline $\begin{array}{l}\text { U Myint } \\
\text { Swe }\end{array}$ & 16 & $0.017 *$ & 21 & $0.001 * * *$ & 19 & $0.002 * *$ & 56 & $0.001 * * *$ \\
\hline Karenina & 23 & $0.001 * * *$ & 19 & $0.002 * *$ & 16 & $0.017 *$ & 58 & $0.001 * * *$ \\
\hline Hmawe Ni & 16 & $0.017^{* *}$ & 14 & 0.017 & 16 & $0.017^{* *}$ & 46 & $0.001 * * *$ \\
\hline Chloe & 14 & 0.017 & 15 & $0.001 * * *$ & 16 & $0.017 * *$ & 45 & $0.001 * * *$ \\
\hline Domino & 11 & 0.001 & 11 & 0.001 & 14 & 0.017 & 36 & 0.017 \\
\hline Tuk & 15 & $0.001 * * *$ & 12 & 0.263 & 16 & $0.017^{* *}$ & 43 & $0.003 * *$ \\
\hline
\end{tabular}

944

956

957

958

959

960

961

962

963

964

965

966

967

968

969

970

971

972

973

974

Across each set (maximum correct is 30 trials per set), and in total (maximum score is 90 trials) and binomial test results. Values noted as significant represent performance above chance level. $P$ values that are significant but not annotated represent performance below chance levels.

*significant at the 0.05 level

**significant at the 0.01 level

***significant at the 0.001 level 
D’Agostino and Cunningham 40

975 Table V: Chi square tests to evaluate individual positional bias on experimental trials of 976 Experiment 2.

977

\begin{tabular}{lcccccccc}
\hline \multicolumn{1}{c}{ Subject } & \multicolumn{2}{c}{ S1 } & \multicolumn{2}{c}{ S2 } & \multicolumn{2}{c}{ S3 } & \multicolumn{2}{c}{ Total } \\
\hline & $\chi^{2}(\mathbf{2})$ & $\boldsymbol{p}$ & $\chi^{2}(\mathbf{2})$ & $\boldsymbol{p}$ & $\chi^{2}(\mathbf{2})$ & $\boldsymbol{p}$ & $\chi^{2}(\mathbf{2})$ & $\boldsymbol{p}$ \\
\hline Marlow & 4.200 & 0.122 & 6.200 & $0.045^{*}$ & 4.200 & 0.122 & 14.067 & $0.001^{* * *}$ \\
Khin Maung & 4.200 & 0.122 & 3.800 & 0.150 & 1.800 & 0.407 & 4.067 & 0.131 \\
Win & & & & & & & & \\
U Myint Swe & 9.800 & $0.007^{* *}$ & 7.800 & $0.020^{*}$ & 9.800 & $0.007^{* *}$ & 26.467 & $0.001^{* * *}$ \\
Karenina & 0.200 & 0.905 & 3.800 & 0.150 & 15.800 & $0.001^{* * *}$ & 13.867 & $0.001^{* * *}$ \\
Hmawe Ni & 3.800 & 0.150 & 4.200 & 0.122 & 32.00 & 0.202 & 9.624 & $0.008^{*}$ \\
Chloe & 0.600 & 0.439 & 0.133 & 0.715 & 13.400 & $0.001^{* * *}$ & 13.867 & $0.001^{* * *}$ \\
Domino & 6.200 & $0.045^{*}$ & $-{ }^{a}$ & $-{ }^{a}$ & 2.600 & 0.273 & 4.467 & 0.107 \\
Tuk & 1.400 & 0.497 & 11.400 & $0.003^{* *}$ & 3.800 & 0.150 & 10.400 & $0.006^{* *}$ \\
\hline
\end{tabular}

Significant values denote a deviance from the expected values of 10 choices at each location. For 980 all gibbons with a bias, it was for the center door.

981

$982{ }^{a}$ observed and expected values were the same therefore Chi square was not calculated

$983 *$ significant at the 0.05 level

$984 * *$ significant at the 0.01 level

$985 * * *$ significant at the 0.001 level 
Figure 1. Betty (Female H. leuconedys) engaged in Experiment 1. The gibbons reached through the fencing and flipped open the buckets to reveal the food items. The buckets were drilled in place with hinges that allowed them to be easily flipped and replaced to the different sets.

$152 \times 86 \mathrm{~mm}(220 \times 220$ DPI $)$ 
Figure 2. Feeding platform used for Experiment 2. This platform required the gibbons to reach through doors to select the hidden food item. Three laminated color boards were inserted onto the front of the feeding platform behind a Plexiglas screen. $246 \times 166 \mathrm{~mm}(72 \times 72$ DPI) 
Figure 3: Adjusted means for number of trials needed to reach criterion performance (10 consecutive first choices of preferred food type) by genus (a) and percentage of correct responses by genus in the postcriterion phase $215 \times 279 \mathrm{~mm}(200 \times 200 \mathrm{DPI})$ 


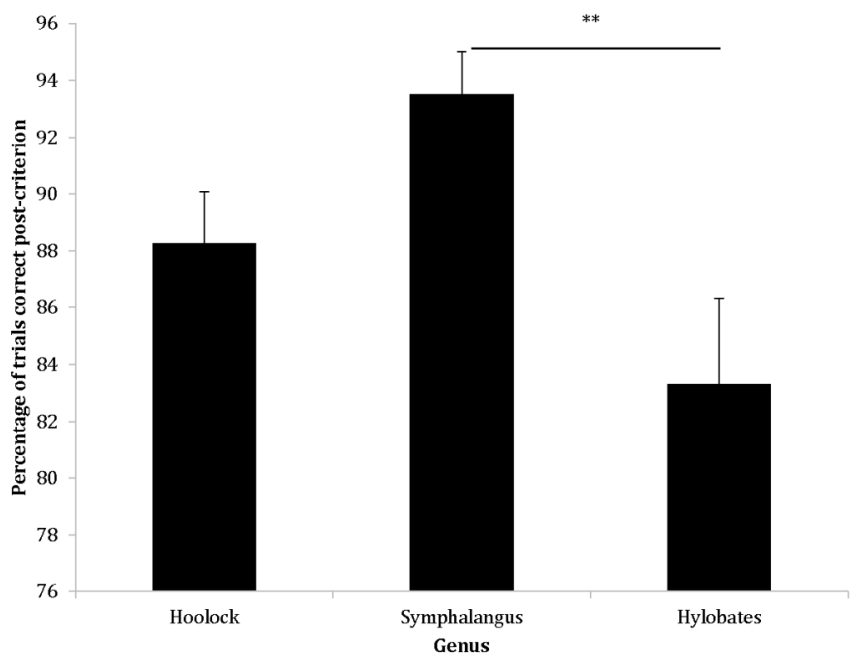

(b). Error bars represent $\pm 1 \mathrm{SE} . * *$ denotes significance at $\mathrm{p}<0.001$ $215 \times 279 \mathrm{~mm}(200 \times 200$ DPI $)$ 




Figure 4: Adjusted means for number of trials to reach criterion performance in the learning trials of Experiment 2 by genus. Error bars represent $\pm 1 \mathrm{SE} . * *$ denotes significance at the 0.001 level. $215 \times 279 \mathrm{~mm}(200 \times 200 \mathrm{DPI})$ 